\title{
Convergent evidence for the antiviral effects of several FDA-approved phenothiazine antipsychotics against SARS-CoV-2 and other coronaviruses
}

\author{
Rodrigo Machado-Vieira, ${ }^{1,2}$ (iD João Quevedo, ${ }^{2,3,4,5}$ (iD Lokesh Shahani, ${ }^{2}$ (iD Jair C. Soares ${ }^{1,2,4}$ (iD) \\ ${ }^{1}$ Experimental Therapeutics and Molecular Pathophysiology Program, Faillace Department of Psychiatry and Behavioral Sciences, McGovern \\ Medical School, The University of Texas Health Science Center at Houston (UTHealth), Houston, TX, USA. ${ }^{2}$ Center of Excellence in Mood \\ Disorders, Faillace Department of Psychiatry and Behavioral Sciences, McGovern Medical School, UTHealth, Houston, TX, USA. \\ ${ }^{3}$ Translational Psychiatry Program, Faillace Department of Psychiatry and Behavioral Sciences, McGovern Medical School, UTHealth, \\ Houston, TX, USA. ${ }^{4}$ Neuroscience Graduate Program, The University of Texas MD Anderson Cancer Center, UTHealth Graduate School of \\ Biomedical Sciences, Houston, TX, USA. ${ }^{5}$ Programa de Pós-Graduação em Ciências da Saúde, Universidade do Extremo Sul Catarinense \\ (UNESC), Criciúma, SC, Brazil.
}

There is an urgent need to identify rapidly accessible and effective medications to treat coronavirus disease 2019 (COVID-19). At present, this is undoubtedly the most important global priority in clinical research. The most obvious drugs to repurpose against the new coronavirus have been those agents effective against other viruses. However, to date, these agents have not shown conclusive evidence of effectiveness. Thus, the systematic screening and validation of alternative repurposed drugs for COVID-19, using in vitro and in vivo models, may provide evidence to rapidly identify new candidates with potential antiviral efficacy against SARS-CoV-2 for further testing.

A previous screen evaluating thousands of FDAapproved drugs for antiviral activity against two other highly pathogenic human coronaviruses, SARS-CoV and MERS-CoV, was performed years ago. This study found 27 drugs that inhibited the replication of SARS-CoV and MERS-CoV in vitro, ${ }^{1}$ belonging to 13 different classes of pharmaceuticals.

Twenty FDA-approved candidate drugs showing antiviral effects against SARS-CoV and MERS-CoV in the initial study ${ }^{1}$ were retested in vitro against the new SARSCoV-2. ${ }^{2}$ Of these, 17 also displayed significant antiviral activity, inhibiting SARS-CoV-2 at a safe (non-cytotoxic) range of IC50 values, ${ }^{2}$ i.e., these 17 drugs targeted, in a convergent manner, more than one human coronavirus type.

From these previous studies, we observed that, out of the 17 drugs showing inhibition of SARS-CoV-2 viral activity, four $(23.5 \%)$ agents (promethazine, fluphenazine, chlorpromazine, and thiethylperazine) belong to the phenothiazine class, which is widely used in psychiatry and other areas of medicine. This observation was not reported in the

Correspondence: Rodrigo Machado-Vieira, The University of Texas Health Science Center at Houston (UTHealth), McGovern Medical School, Faillace Department of Psychiatry and Behavioral Sciences, 1941 East Road, Houston, TX 77054, USA.

E-mail: Rodrigo.MachadoVieira@uth.tmc.edu

Submitted Dec 04 2020, accepted Dec 04 2020, Epub Jan 112021. original study. Among these four phenothiazines, only chlorpromazine was further studied, with additional positive findings related to inhibition of cytopathic effect (CPE), mRNA synthesis, and SARS-CoV-2 viral particle production. ${ }^{2}$

Recently, the National Center for Advancing Translational Research (NCATS), affiliated with the National Institutes of Health (NIH), made available the COVID-19 OpenData Portal to openly and quickly share drug repurposing data testing for antiviral activity against SARS-CoV-2. ${ }^{3}$ Nearly 3,000 approved drugs have been tested and data obtained based on NCATS-validated SARS-CoV-2 assays (latest update in September 2020).

In the present report, we provide the first convergent evidence from two validated datasets evaluating antiviral effects against different human coronavirus types, with a particular focus on SARS-CoV-2. We reviewed the NCATS approved drug collection (NPC) (2,677 drugs total, any formulation). We selected as criteria the presence of one or more parameters with high-quality evidence as well as at least 3 (out of 4) key parameters with any level of evidence (Table 1). We hypothesized that antipsychotic drugs sharing the three-ring structure of the phenothiazine class, with different side chains, would display a unique pharmacological profile to convergently inhibit SARS-CoV-2.

Parameters included the surface ACE2 receptor protein, which has been shown to be the primary host factor recognized and targeted by SARS-CoV-2 virions. Disrupting the spike protein-ACE2 interaction may limit the ability of SARS-CoV-2 virions to infect the host. Two specific ACE2-related parameters were evaluated: spikeACE2 protein-protein interaction (AlphaLISA-1) and spike-ACE2 protein-protein interaction (TruHit Counterscreen-2). ${ }^{4}$ In terms of live virus infectivity, two other parameters were included: SARS-CoV-2 CPE, a live

How to cite this article: Machado-Vieira R, Quevedo J, Shahani L, Soares JC. Convergent evidence for the antiviral effects of several FDA-approved phenothiazine antipsychotics against SARS-CoV-2 and other coronaviruses. Braz J Psychiatry. 2021;43:462-464. http:// dx.doi.org/10.1590/1516-4446-2020-0024 
Table 1 Effects of multiple phenothiazines matching criteria for antiviral effects against SARS-CoV-2

\begin{tabular}{|c|c|c|c|c|c|}
\hline \multirow[b]{2}{*}{ Drug } & \multirow[b]{2}{*}{$\begin{array}{l}\text { Antiviral } \\
\text { criteria* }\end{array}$} & \multicolumn{2}{|c|}{ Viral entry } & \multicolumn{2}{|c|}{ Live virus infectivity } \\
\hline & & $\begin{array}{l}\text { Spike-ACE2 protein-protein } \\
\text { interaction (AlphaLISA) }\end{array}$ & $\begin{array}{c}\text { Spike-ACE2 protein-protein } \\
\text { interaction } \\
\text { (TruHit Counterscreen) }\end{array}$ & $\begin{array}{l}\text { Cytopathic effect } \\
\text { (CPE) }\end{array}$ & $\begin{array}{l}\text { Host tox } \\
\text { counterscreen }\end{array}$ \\
\hline Fluphenazine & Yes & ++ & ++ & + & + \\
\hline Chlorpromazine & Yes & + & ++ & + & + \\
\hline Thiethylperazine & Yes & ++ & ++ & & + \\
\hline Promethazine & Yes & ++ & + & + & \\
\hline Perphenazine & Yes & ++ & ++ & + & \\
\hline Thioproperazine & Yes & ++ & ++ & + & \\
\hline Periciazine & Yes & + & ++ & + & \\
\hline Trifluoperazine & Yes & + & ++ & + & + \\
\hline Triflupromazine & Yes & + & ++ & + & + \\
\hline Thioridazine & Yes & ++ & ++ & & + \\
\hline
\end{tabular}

Any evidence: yes or no; if yes, quality of evidence: + low; ++ high.

Bold type indicates phenothiazines that also showed significant antiviral effects against other coronaviruses in previous datasets.

* Selected drug has at least high-quality evidence for one parameter and any level of evidence for at least three or four parameters.

SARS-CoV-2 virus assay, measured the ability of compounds to reverse the viral-induced CPE in Vero E6 host cells; a similar parameter was the host tox counter screen, a cell-based assay measuring host cell ATP content as a readout for cytotoxicity in Vero E6 cells selected for high ACE2 expression. ${ }^{5}$

The results are presented in Table 1 (findings reflect the NCATS dataset available in September 2020). We found a total of 137 FDA-approved drugs matching the predefined criteria for antiviral effects against SARS-CoV2. We also evaluated whether any specific pharmacological class would contain a cluster of agents displaying significant antiviral activity. It was observed that only the phenothiazine class contained at least 10 drugs matching criteria for antiviral effects against SARS-CoV-2. Exactly 10 phenothiazines $(7.3 \%$ of the total, see Table 1$)$ matched the criteria for anti-SARS-CoV-2 effects.

This list includes chlorpromazine, which had been recently highlighted based on preliminary positive results. ${ }^{2,6}$ Fluphenazine also showed antiviral effects against SARSCoV, MERS-CoV, and SARS-CoV-2 in previous studies, ${ }^{1,2}$ which was confirmed in the present study (showing slight superiority over chlorpromazine). Thiethylperazine and promethazine, the two other drugs (out of 17) that had shown antiviral effects against SARS-CoV and MERSCoV in vitro, ${ }^{1,2}$ also matched criteria for antiviral effects against SARS-CoV-2 in our study (Table 1).

Other phenothiazines matching the criteria were thioridazine, perphenazine, thioproperazine, periciazine, trifluoperazine, and triflupromazine. Strikingly, no other pharmacological class showed similar results in terms of the absolute or relative number of agents with such activity.

This is the first description of phenothiazines as a class with potential utility in the treatment of COVID-19 based on in vitro studies. The present report provides evidence supporting convergent in vitro antiviral effects against SARS-CoV-2 of a cluster of drugs belonging to the same pharmacological class (with a phenothiazine skeleton). Interestingly, besides their efficacy as antipsychotics (and antiemetics in certain cases), this class of agents has also shown antiviral, antibacterial, and anti-prion activities in previous in vitro studies (reviewed in Varga et al. ${ }^{7}$ ).

Phenothiazines have good overall distribution throughout the body, including the lungs, and cross the bloodbrain barrier, which could eventually help to prevent psychiatric and neurological consequences of COVID-19. The potential antiviral effects of phenothiazines have been associated with their ability to inhibit viral binding to plasma membrane receptors, thus blocking endocytosis and inhibiting the required DNA replication. The few early studies on the role of phenothiazines only focused on the antiviral activity of chlorpromazine, ${ }^{7}$ while other similar agents described herein were not studied further regarding their potential antiviral effects against human coronaviruses.

We conclude that several typical antipsychotics sharing the same phenothiazine skeleton may have antiviral effects against SARS-CoV-2. Further confirmatory in vivo and epidemiological studies are warranted.

\section{Acknowledgements}

The NCATS allowed access to the dataset from which the results were obtained.

\section{Disclosure}

RM-V has received consulting fees from Eurofarma Pharmaceuticals and BioStrategies Group; has research contracts with Janssen Pharmaceuticals; has received speaker fees from Otsuka and Cristalia; is a member of the scientific board of Symbinas Pharmaceuticals; and receives copyright from Elsevier/Academic Press. JQ has received clinical research support from LivaNova; has been in the speaker bureau for Myriad Neuroscience, Janssen Pharmaceuticals, and Abbvie; is a partner at Instituto de Neurociências Dr. Joao Quevedo; and receives copyright from Artmed Editora, Artmed Panamericana, and Elsevier/Academic Press. The other authors report no conflicts of interest. 


\section{References}

1 Dyall J, Coleman CM, Hart BJ, Venkataraman T, Holbrook MR, Kindrachuk J, et al. Repurposing of clinically developed drugs for treatment of Middle East respiratory syndrome coronavirus infection. Antimicrob Agents Chemother. 2014;58:4885-93.

2 Weston S, Coleman CM, Haupt R, Logue J, Matthews K, Frieman MB. Broad anti-coronaviral activity of FDA approved drugs against SARS-CoV-2 in vitro and SARS-CoV in vivo. bioRxiv. 2020. doi: $10.1101 / 2020.03 .25 .008482$

3 National Center for Advancing Translational Sciences (NCATS). OpenDataPortal. OpenData COVID-19 [Internet]. 2020 [cited 2020 Dec 10]. opendata.ncats.nih.gov/covid19/

4 Zhang $\mathrm{H}$, Penninger JM, Li Y, Zhong N, Slutsky AS. Angiotensinconverting enzyme 2 (ACE2) as a SARS-CoV-2 receptor: molecular mechanisms and potential therapeutic target. Intensive Care Med. 2020;46:586-90.

5 Gorshkov K, Chen CZ, Bostwick R, Rasmussen L, Xu M, Pradhan M, et al. The SARS-CoV-2 cytopathic effect isblocked with autophagy modulators. Version 2. bioRxiv. Preprint. 2020 May 28. doi: 10.1101/ 2020.05.16.091520.

6 Plaze M, Attali D, Prot M, Petit AC, Blatzer M, Vinckier F, et al. Inhibition of the replication of SARS-CoV-2 in human cells by the FDA-approved drug chlorpromazine. bioRxiv. 2020. doi: 10.1101/ 2020.05.05.079608

7 Varga B, Csonka Á, Csonka A, Molnár J, Amaral L, Spengler G. Possible biological and clinical applications of phenothiazines. Anticancer Res. 2017;37:5983-93. 